\title{
Advances in the reconstruction of LBT LINC-NIRVANA images
}

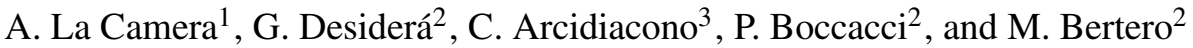 \\ 1 INAF - Osservatorio Astrofisico di Arcetri, Lgo. E. Fermi 5, 50125 Firenze, Italy \\ e-mail: bertero@disi.unige.it \\ 2 DISI, Università di Genova, Via Dodecaneso 35, 16146 Genova, Italy \\ 3 INAF - Osservatorio Astronomico di Padova, Vicolo dell'Osservatorio 5, 35122 Padova, Italy
}

Received 1 March 2007 / Accepted 15 May 2007

\section{ABSTRACT}

\begin{abstract}
Context. LINC-NIRVANA, the Fizeau interferometer of the Large Binocular Telescope (LBT), will require routine use of image reconstruction methods for data reduction. To this purpose our group has already developed the software package AIRY (Astronomical Image Restoration in interferometrY).

Aims. Observations of a target, with different orientations of the baseline of LINC-NIRVANA, will provide images with different orientations with respect to the CCD camera. This rotation effect was not taken into account in our previous work. Therefore in this paper we propose a method able to compensate for the rotation of the field of view. Moreover we investigate acceleration techniques for reducing the computational burden of multiple image deconvolution.

Methods. The basic method is a suitable modification of the Richardson-Lucy algorithm, also implementing an approach we proposed for reducing boundary effects. Acceleration techniques, proposed by Biggs \& Andrews, are extended and applied to this new algorithm. Finally a method for estimating the unknown point spread function (PSF) by extracting and extrapolating the image of a reference star is developed and implemented.

Results. The method introduced for compensating object rotation and reducing boundary effects, as well as its accelerated versions, are tested on simulated LINC-NIRVANA images, using the VLT image of the Crab Nebula as test object. The results are very promising. Moreover the method for PSFs extraction is tested on simulated images, derived from the LBT image of the galaxy NGC 6946 and obtained by convolving this image with PSFs computed by means of the numerical code LOST (Layer Oriented Simulation Tool).
\end{abstract}

Key words. techniques: interferometric - thechniques: image processing - methods: data analysis - methods: numercial

\section{Introduction}

In previous papers (Bertero \& Boccacci 2000; Correia et al. 2002; Carbillet et al. 2002; Anconelli et al. 2005), we developed methods and software for the deconvolution of multiple interferometric images of the same astronomical target. One of the results is the software package AIRY (Astronomical Image Restoration in interferometrY), and version 3.0 is now available (see http://dirac.disi.unige.it and http://www-luan. unice.fr/caos). This tool, described in Correia et al. (2002), can be applied to Fizeau interferometers, such as the LINC-NIRVANA (Lbt INterferometric Camera and Near-InfraRed/Visible Adaptive iNterferometer for Astronomy) (http://www.mpia.de/LINC/), the GermanItalian beam combiner for the Large Binocular Telescope (LBT). Indeed, LINC-NIRVANA (in the following denoted by LN) will routinely require the use of image reconstruction algorithms (Hofmann et al. 2005). The LBT (http://lbtwww.arcetri.astro.it), under completion on Mount Graham, Arizona, will consist of two $8.4 \mathrm{~m}$ mirrors on a common mount, with a spacing of $14.4 \mathrm{~m}$ between their centres, so that a maximum baseline of $22.8 \mathrm{~m}$ will be available. The two primary mirrors have been already installed and aluminized; "first light" was achieved on October 12, 2005.

We have already developed a number of iterative reconstruction methods for LN imaging, mainly derived from suitable modifications of the Richardson-Lucy method (RLM), equipped with acceleration techniques, based, for instance, on the ordered subset expectation maximization approach (OSEM: Hudson \& Larkin 1994) and on the multiplicative relaxation method (Iusem 1991). Moreover, the split gradient method (SGM; Lanteri et al. 2002) is used for implementing different kinds of regularization. A survey is found in Bertero et al. (2006). However, in all these methods we assume that the camera rotates in such a way that the position of the object is fixed inside its field of view (FoV). This will not the case when LN is operating; therefore, in this paper, we introduce a modification of our methods compensating for this effect.

The approach we propose is described in Sect. 2 and is computationally very expensive since it requires the immersion of the detected images into broader arrays. For instance, for a $256 \times 256$ image, the computational time on a good PC can range from a few minutes to one hour, depending on the SNR (higher SNR implies a larger number of iterations). Since the images of the LN-camera will be $2048 \times 2048$, it is clear that computational efficiency can be an important issue. Therefore in this paper we also extend the acceleration methods introduced by Biggs \& Andrews $(1997,1998)$ to our approach, and we investigate their effect. The preliminary results obtained with these methods are promising since they are effective when the original method requires a very large number of iterations. An average gain by a factor of 10 seems to be feasible. These results are described in Sect. 3.

Another important issue for the reconstruction of the detected images is the estimation of the PSFs. Since they depend on the adaptive optics (AO) correction achieved in that 
particular observation, it is obvious that they cannot be modeled and computed but must be extracted from the image of one or more reference stars. Therefore, in Sect. 4 we propose a method for extracting and extrapolating the PSFs from the detected images, and we test this method on simulated images.

\section{Compensation for object rotation}

We assume the model of image formation described in the previous papers. We use bold letters for denoting $N \times N$ arrays, whose pixels are indexed by a multi-index $\boldsymbol{n}=\left\{n_{1}, n_{2}\right\}$. Moreover, we assume that we have $p$ images acquired with $\mathrm{LN}$, corresponding to $p$ different orientations of the baseline and denoted by $\boldsymbol{g}_{1}, \boldsymbol{g}_{2}, \ldots, \boldsymbol{g}_{p}$.

Since the camera is rotating with the baseline, the fringes of the PSFs always have the same orientation (for instance, the vertical one) in the image array, so that the $p$ different images correspond to $p$ rotated versions of the observed object. If this is completely contained in the FoV of the camera and is surrounded by empty sky, then we only need to anti-rotate the $p$ images in order to align the different versions of the object. However, if the object is broader than the FoV (10" in the case of LN-camera), then parts of the object present in one image may not be present in another one. In such a case it may be convenient to reconstruct only the part of the object that is common to all images.

We formalize the previous statement. We use again the notation $\boldsymbol{g}_{1}, \boldsymbol{g}_{2}, \ldots, \boldsymbol{g}_{p}$ for denoting now those images that have been obtained by anti-rotating the detected ones (using, for instance, a standard routine for image rotation); they are immersed into broader arrays, let us say $2 N \times 2 N$, that are obtained by zeropadding. We denote the set of the multi-index values corresponding to the broader array by $\bar{S}$ and by $S_{j} \subset \bar{S}$ the set of the values of the multi-index corresponding to the $j$ th image. Then we denote the intersection of the sets $S_{j}$ by $S$; it will correspond, in general, to a polygonal domain interior to the square corresponding to $\bar{S}$.

The approach consists in extracting the part corresponding to the domain $S$ from each image and to extend the result to $\bar{S}$ by zero padding; next, these images are deconvolved by the method we proposed for the reduction of boundary effects (Bertero \& Boccacci 2005; Anconelli et al. 2006). Indeed this method can be easily extended to the case where the image domain is not a square. For the convenience of the reader, we recall the basic points of the method. A more complete description is found in Anconelli et al. (2006).

We denote by $\boldsymbol{K}_{j}$ the PSF, defined over $\bar{S}$ and corresponding to the anti-rotated image $\boldsymbol{g}_{j}$; this PSF can be obtained, for instance, by the method described in Sect. 4. Then, the first step is to define the reconstruction domain $R$ that is broader than the image domain $S$. To this purpose we introduce the weighting functions, defined over $\bar{S}$,

$$
\begin{aligned}
\alpha_{j}(\boldsymbol{n}) & =\left(\boldsymbol{K}_{j}^{T} * \boldsymbol{M}_{S}\right)(\boldsymbol{n}), \\
\alpha(\boldsymbol{n}) & =\frac{1}{p} \sum_{j=1}^{p} \alpha_{j}(\boldsymbol{n}),
\end{aligned}
$$

where $\boldsymbol{K}_{j}^{T}(\boldsymbol{n})=\boldsymbol{K}_{j}(-\boldsymbol{n})$, and $\boldsymbol{M}_{S}(\boldsymbol{n})$ is the mask of the image domain. Given a thresholding parameter $\sigma<1$, the reconstruction domain is defined by

$R=\{\boldsymbol{n} \mid \alpha(\boldsymbol{n})>\sigma\}$.

The next step is to define the normalization condition that must be satisfied by any estimate $f$ of the unknown object. Since we assume that the background $\boldsymbol{b}_{j}$ of the image $\boldsymbol{g}_{j}$ is known, the normalization condition is given by

$$
\sum_{\boldsymbol{n} \in R} \alpha(\boldsymbol{n}) \boldsymbol{f}(\boldsymbol{n})=\frac{1}{p} \sum_{j=1}^{p} \sum_{\boldsymbol{n} \in S}\left\{\boldsymbol{g}_{j}(\boldsymbol{n})-\boldsymbol{b}_{j}\right\} \doteq c .
$$

Finally, the last ingredient is provided by the window functions to be inserted into our version of RLM. They are given by

$\boldsymbol{w}_{j}(\boldsymbol{n})=\left\{\begin{array}{cl}\frac{1}{\alpha_{j}(\boldsymbol{n})}, & \text { if } \boldsymbol{n} \in R, \\ 0, & \text { otherwise. }\end{array}\right.$

With all the quantities introduced above and using the notations $A_{j} \boldsymbol{f}=\boldsymbol{K}_{j} * \boldsymbol{f}, A_{j}^{T} \boldsymbol{f}=\boldsymbol{K}_{j}^{T} * \boldsymbol{f}$, the version of the OSEM algorithm, with correction of both rotation and boundary effects, is as follows.

- Initialize the algorithm with $\boldsymbol{f}^{(0)}=$ const., satisfying the normalization condition of Eq. (3).

- Given $\boldsymbol{f}^{(k)}$, set $\boldsymbol{h}^{(0)}=\boldsymbol{f}^{(k)}$ and, for $j=1, \ldots, p$, compute

$$
\begin{aligned}
\tilde{\boldsymbol{h}}^{(j)} & =\boldsymbol{w}_{j} \boldsymbol{h}^{(j-1)}\left(A_{j}^{T} \frac{\boldsymbol{g}_{j}}{A_{j} \boldsymbol{h}^{(j-1)}+\boldsymbol{b}_{j}}\right), \\
\tilde{\boldsymbol{c}}^{(j)} & =\sum_{\boldsymbol{n} \in R} \alpha(\boldsymbol{n}) \tilde{\boldsymbol{h}}^{(j)}(\boldsymbol{n}), \boldsymbol{h}^{(j)}=\frac{c}{\tilde{\boldsymbol{c}}^{(j)}} \tilde{\boldsymbol{h}}^{(j)} .
\end{aligned}
$$

- Set $\boldsymbol{f}^{(k+1)}=\boldsymbol{h}^{(p)}$.

We have verified the numerical accuracy of the algorithm by taking the $512 \times 512$ VLT image of the Crab nebula as our test object. First the object has been rotated clockwise by $60^{\circ}$ and $120^{\circ}$ using the routine ROT of the IDL library. The three objects, immersed into an array $1024 \times 1024$ are shown in the left panels of Fig. 1. Next we have assumed observation in $K$-band, with an integrated magnitude of the object of 10 . The pixel size is that of LN, i.e. 5.12 mas. The three images have been convolved with an ideal interferometric PSF, with vertical fringes. Moreover, the following parameters were assumed in image generation: an integration time of $20 \mathrm{~min}$ for each image, a telescope surface of $104 \mathrm{~m}^{2}$, a sky background of $12.5 \mathrm{mag} / \mathrm{arcsec}^{2}$, and an efficiency of $30 \%$. The results were perturbed with Poisson noise and a read-out-noise (RON) of $10 \mathrm{e}^{-} \mathrm{rms}$ and are shown in the right panels of Fig. 1.

From these images we extracted the central parts $256 \times 256$ and these have been rotated counter-clockwise by the corresponding angles. The intersection of the three rotated images, i.e. the domain $S$, is a dodecagonal domain. The region of the object corresponding to this domain and immersed in a $512 \times 512$ array is shown in the upper-left panel of Fig. 2.

The three images have been deconvolved by means of the algorithm described above, using rotated ideal PSFs. In view of a comparison with the acceleration methods described in the next section, we have computed at each iteration a relative rms error defined as the ratio between the Euclidean norm of $\boldsymbol{f}^{(k)}-\boldsymbol{f}$ and the Euclidean norm of $\boldsymbol{f}$

$\rho^{(k)}=\frac{\left\|\boldsymbol{f}^{(k)}-\boldsymbol{f}\right\|}{\|\boldsymbol{f}\|}$

This error reaches a minimum of about $6.7 \%$ after about 2300 iterations (the minimum, however, is very flat). The corresponding reconstructed image is shown in the upper-right panel of Fig. 2. We point out that this error is a global one providing an estimate of the average accuracy reached by the reconstruction method. 

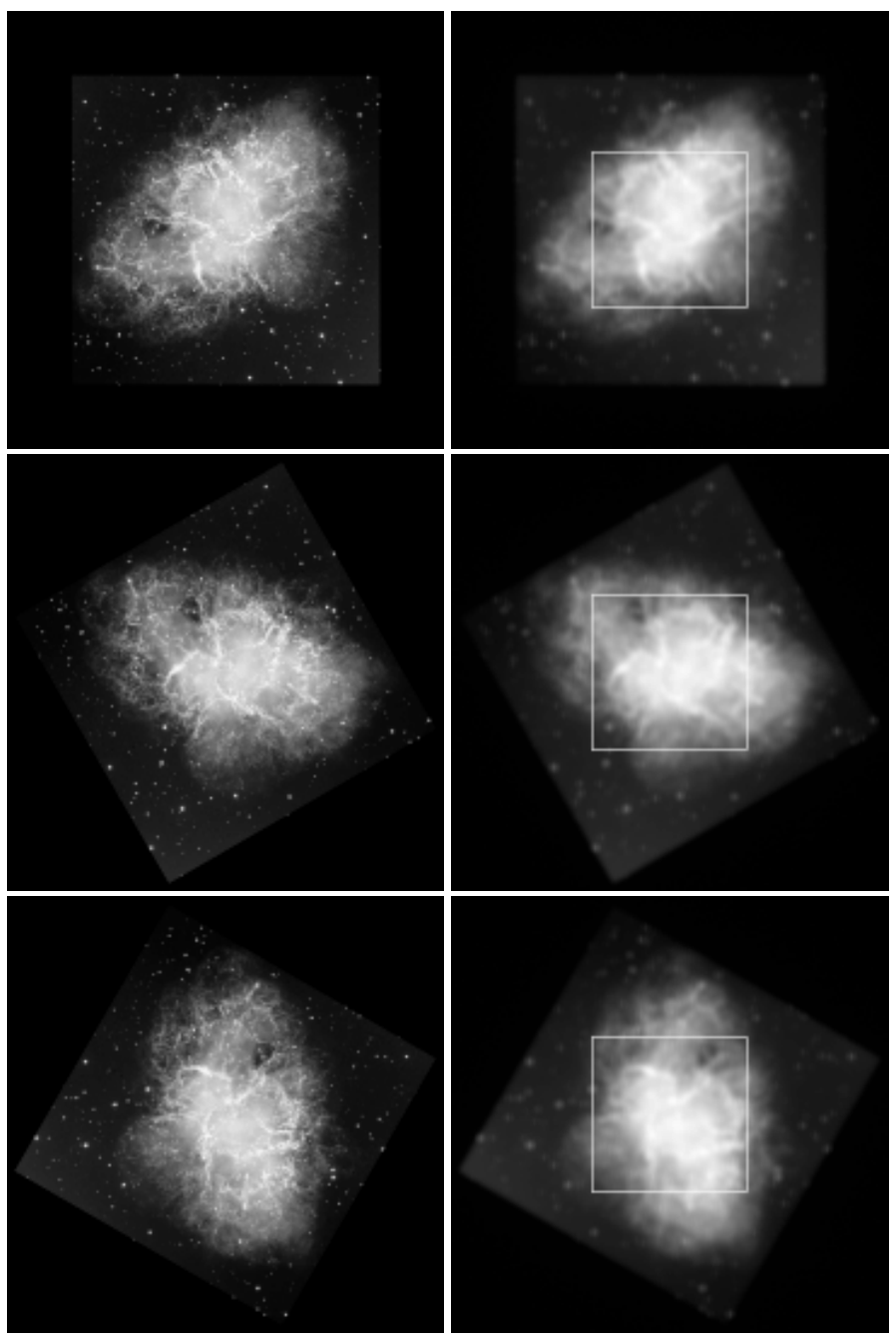

Fig. 1. Left panels: the three rotated objects obtained from the VLT image of the Crab Nebula. Right panels: the corresponding interferometric images obtained by convolving the object of the left panel with an ideal interferometric PSF with vertical fringes. The squares indicate the images that have been extracted for deconvolution.

A more accurate analysis should require an evaluation of the accuracy of astronomical parameters such as photometry, astrometry, or others. We also note that, if we increase the number of the detected images (for instance six equispaced images instead of three), we do not get a significant reduction of the rms error defined above. This result, however, holds true in this specific example because we use ideal interferometric PSFs: three equispaced orientations are enough to provide good coverage of the $u, v$-plane in such a case. In the case of AO-corrected PSFs with a relatively low SR it may be necessary to use a larger number of orientations. For instance, in Sect. 4 we use six equispaced orientations in order to reduce artifacts in the reconstructed image.

Finally, to check the statistical accuracy of the result, we computed the normalized residuals

$\mathbf{R}_{j}(\boldsymbol{n})=\frac{\boldsymbol{g}_{j}(\boldsymbol{n})-\left[\left(A_{j} \boldsymbol{f}\right)(\boldsymbol{n})+\boldsymbol{b}_{j}\right]}{\sqrt{\left(A_{j} \boldsymbol{f}\right)(\boldsymbol{n})+\boldsymbol{b}_{j}}} ; j=1, \ldots, p$.

They were computed only in the central square $128 \times 128$ of the domain $S$, in order to exclude residual boundary effects. In the middle-left panel of Fig. 2 we find what is obtained when $f$ is the original image of the Crab nebula (we give the result only in the case of the image corresponding to $0^{\circ}$ ). The distribution
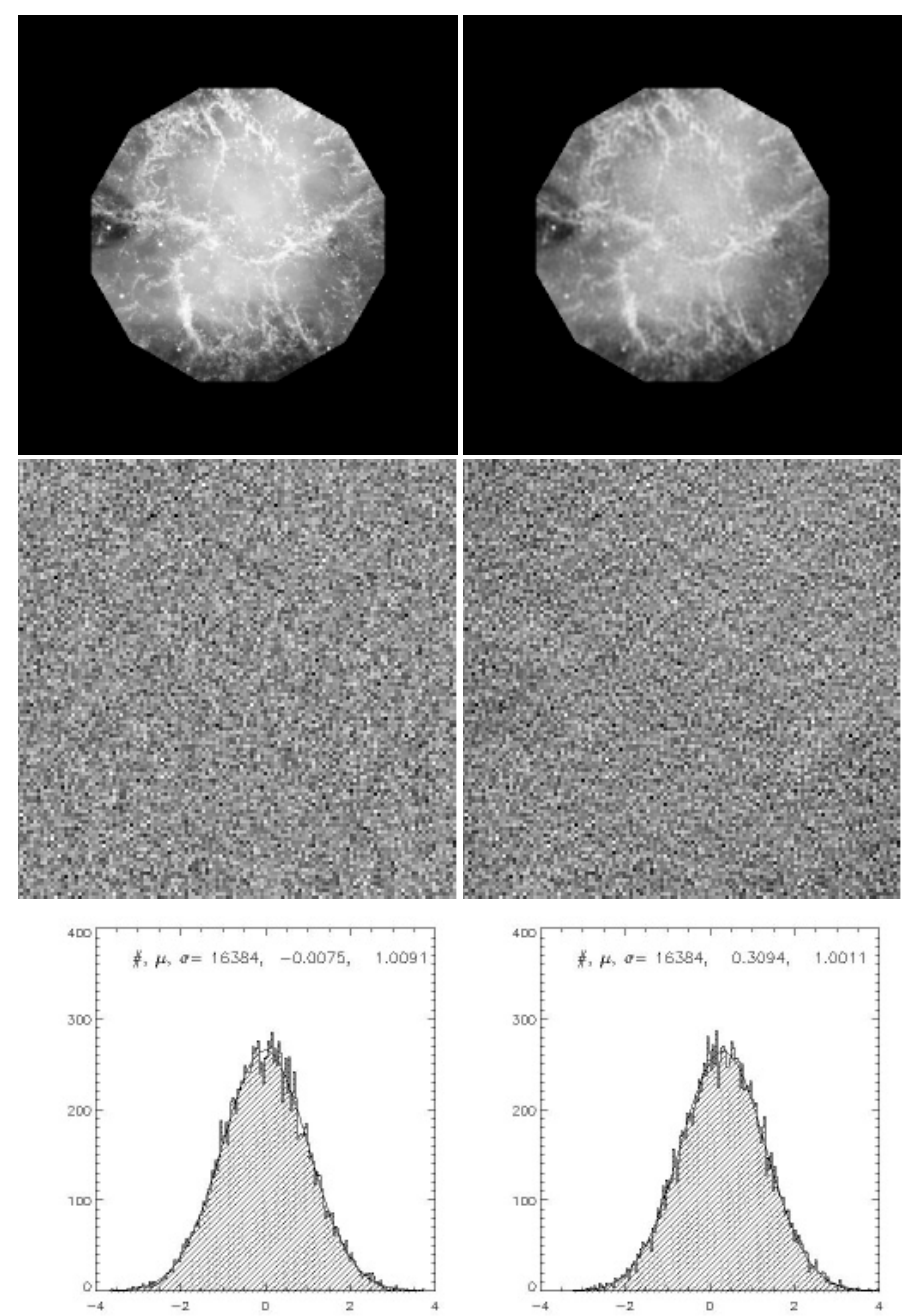

Fig. 2. Upper-left panel: the object to be reconstructed, restricted to the domain $S$. Upper-right panel: the reconstruction provided by the method described in the text. Middle-left panel: the normalized residual of the object. Middle-right panel: the normalized residual of the reconstruction. Lower-left panel: the histogram of the values of the normalized residual of the object. Lower-right panel: the histogram of the values of the normalized residual of the reconstruction.

of the pixel values is a Gaussian with mean -0.01 and $\sigma=1.01$ and is plotted in the lower-left panel. In the middle-right panel of the same figure we give the normalized residual corresponding to the reconstructed object. The distribution of the pixel values is again a Gaussian with mean +0.31 and $\sigma=1.00$ and is plotted in the lower-right panel. We think that the agreement is fairly good.

\section{Acceleration methods}

An acceleration scheme for iterative methods has been proposed by Biggs \& Andrews (1997) and applied to Richardson-Lucy, maximum entropy, and Gerchberg-Saxon algorithms. More generally the approach applies to any iterative method that is characterised by a very slow convergence and can be written in the form

$\boldsymbol{f}^{(k+1)}=T\left(\boldsymbol{f}^{(k)}\right)$

with a linear or nonlinear operator $T$ that does not depend on $k$. In our application, the operator $T$ is defined by one OSEM iteration with boundary effect correction, as defined in the previous 
section, i.e. by a cycle over the $p$ images, including the normalization of the result at each step of the cycle.

The basic idea of the method is to attempt an extrapolation of the iterates. We do not repeat the arguments of Biggs \& Andrews, but only give the algorithm, which implies the storage of 4 images. Since the extrapolation is based on the result of the current iteration and of the previous one, it must be initialized with at least two iterations as defined in Eq. (8). An alternative is to apply the acceleration only when the restoration changes slowly between two iterations. The algorithm we implemented follows.

- Initialize the algorithm with $\boldsymbol{f}^{(0)}=$ const. satisfying the normalization condition of Eq. (3).

- Compute and store $f^{(1)}=T\left(f^{(0)}\right)$ and $f^{(2)}=T\left(f^{(1)}\right)$.

- Store $e^{(0)}=\boldsymbol{f}^{(0)}, \boldsymbol{e}^{(1)}=\boldsymbol{f}^{(1)}$.

- For $k=2,3, . .$, given $\left\{\boldsymbol{f}^{(k)}, \boldsymbol{f}^{(k-1)} ; \boldsymbol{e}^{(k-1)}, \boldsymbol{e}^{(k-2)}\right\}$, compute

$$
\begin{aligned}
& \Delta^{(k)}=\boldsymbol{f}^{(k)}-\boldsymbol{e}^{(k-1)}, \boldsymbol{\Delta}^{(k-1)}=\boldsymbol{f}^{(k-1)}-\boldsymbol{e}^{(k-2)}, \\
& \lambda_{k}=\frac{\left(\boldsymbol{\Delta}^{(k)}, \boldsymbol{\Delta}^{(k-1)}\right)}{\left\|\boldsymbol{\Delta}^{(k-1)}\right\|^{2}}, \\
& \boldsymbol{e}^{(k)}=\boldsymbol{f}^{(k)}+\lambda_{k}\left(\boldsymbol{f}^{(k)}-\boldsymbol{f}^{(k-1)}\right) . \\
&- \text { Compute } \boldsymbol{f}^{(k+1)}=T\left(\boldsymbol{e}^{(k)}\right) .
\end{aligned}
$$

In other words, $\boldsymbol{e}^{(k)}$ is the extrapolated image obtained from $\boldsymbol{f}^{(k)}$ and $\boldsymbol{f}^{(k-1)}$, and $\lambda_{k}$ is the extrapolation step. According to Biggs $\&$ Andrews $\lambda_{k}$ must be constrained to values not exceeding 1 , while the extrapolated image $\boldsymbol{e}^{(k)}$ should be made non-negative by setting possibly negative values to zero.

The main difficulty in applying this method to RLM and similar algorithms is that, thanks to their multiplicative structure, zero values in the extrapolated image $\boldsymbol{e}^{(k)}$ propagate through the subsequent iterations and cannot be removed. For this reason, the linear extrapolation described above is replaced in Biggs \& Andrews (1998) by an exponential extrapolation that does not introduce negative values. Again, the algorithm must be initialized with at least two standard iterations. Therefore we give only the extrapolation step:

$$
\begin{aligned}
& \text { - For } k=2,3, \ldots \text { given }\left\{\boldsymbol{f}^{(k)}, \boldsymbol{f}^{(k-1)} ; \boldsymbol{e}^{(k-1)}, \boldsymbol{e}^{(k-2)}\right\}, \text { compute } \\
& \boldsymbol{\Delta}^{(k)}=\frac{\boldsymbol{f}^{(k)}}{\boldsymbol{e}^{(k-1)}}, \boldsymbol{\Delta}^{(k-1)}=\frac{\boldsymbol{f}^{(k-1)}}{\boldsymbol{e}^{(k-2)}}, \\
& \lambda_{k}=\frac{\left(\boldsymbol{f}^{(k)} \ln \Delta^{(k)}, \boldsymbol{f}^{(k-1)} \ln \Delta^{(k-1)}\right)}{\left\|\boldsymbol{f}^{(k-1)} \ln \Delta^{(k-1)}\right\|^{2}}, 0 \leq \lambda_{k} \leq 1, \\
& \boldsymbol{e}^{(k)}=\boldsymbol{f}^{(k)}\left(\frac{\boldsymbol{f}^{(k)}}{\boldsymbol{f}^{(k-1)}}\right)^{\lambda_{k}} \cdot \\
&- \text { Compute } \boldsymbol{f}^{(k+1)}=T\left(\boldsymbol{e}^{(k)}\right) .
\end{aligned}
$$

Both methods have been applied to the iterative algorithm introduced in the previous section and have been tested on the numerical example described there. Both methods have provided approximately the same acceleration. At each iteration $k$, we computed the relative rms error defined in Eq. (6). In Fig. 3 we give the behaviour of this error (as a function of $k$ ) for the nonaccelerated algorithm, as well as for the two accelerated ones. These algorithms reach about the same minimum value of the relative rms error after 93 iterations. The value is about $6.8 \%$ and is reached by the non-accelerated algorithm after about 900 iterations (we recall that the minimum value is $6.7 \%$ and that the

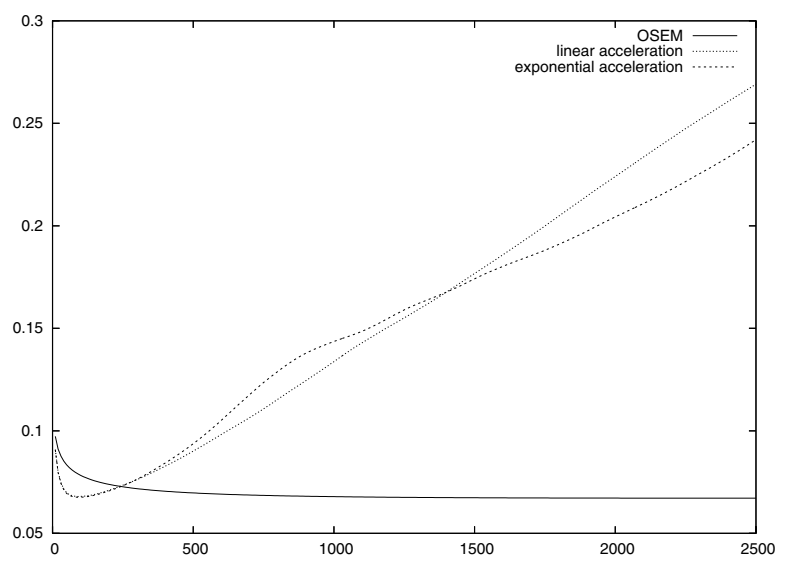

Fig. 3. Behaviour of the reconstruction error as a function of the number of iterations.

minimum is very flat). Therefore there is a gain of about a factor 10 in the number of iterations. However the computational cost of one iteration is not the same in the two cases since, in the accelerated algorithms, it also contains the cost of the extrapolation step. We have verified that, in the case of linear extrapolation, the increase is about $13 \%$, while it is about $30 \%$ in the case of exponential extrapolation. Therefore this preliminary result seems to indicate that the linear extrapolation is more convenient than the exponential one.

Finally, an important point is the fast increase in the reconstruction error after the attainment of the minimum. This fact implies that the choice of the number of iterations is more critical in the case of the accelerated algorithm than in the case of the non-accelerated one. Therefore a stopping rule is needed for application to real images. Unfortunately this is not available, as far as we know; therefore, some practical rule should be derived by means of a suitable training of the algorithm in a simulation program.

\section{PSF extraction and extrapolation}

As is known, the PSF is not known in the case of ground-based telescopes equipped with an adaptive optics (AO) system so it must be obtained from the image of one or more stars contained in the FoV. In this section we describe the method developed for LN images and implemented in a module of the software package AIRY.

A PSF extracted from the image of a star has a much smaller size than the full image. It could be extended by zero padding, but this extension may not be suitable for image deconvolution because this procedure is equivalent to multiplying the full PSF by the window function of the extraction domain; and, in the $u, v$ plane, this is equivalent to convolving the transfer function of the telescope with the Fourier transform of the window function. In other words, a sort of "blurring" is introduced in the Fourier domain. This effect will be evident in the subsequent Fig. 5.

For all these reasons we have implemented an extrapolation of the PSF outside the extraction domain by means of a suitable Lorentzian function. For each selected star in the FoV, our procedure consists of the following steps.

- The centroid of the star is computed and shifted to the centre of the image with a sub-pixel precision.

- A domain of size $2 R \times 2 R$ is extracted from the central region, where $R$ is the extraction radius. This image, which will be denoted by $H(\boldsymbol{n})$, contains not only the image of the star but 
also a background that may be due both to sky emission and to nearby diffuse objects.

- A first estimation of the background is performed with a bilinear function $B_{0}(\boldsymbol{x})=\bar{a}_{0}+\overline{\boldsymbol{a}} \cdot \boldsymbol{x}$, where $\boldsymbol{x}=\left\{x_{1}, x_{2}\right\}$ are coordinates in the image plane and $\overline{\boldsymbol{a}}=\left\{\bar{a}_{1}, \bar{a}_{2}\right\}$ is a pair of constants.

- The image $H(\boldsymbol{n})-\overline{\boldsymbol{a}} \cdot \boldsymbol{n}$ is fitted with a circularly symmetric Lorentzian function superimposed on a constant background

$L_{0}(\boldsymbol{x})=\bar{a}_{0}^{\prime}+\frac{\bar{a}_{3}}{1+\frac{|x|^{2}}{\bar{a}_{4}^{2}}}$.

- The estimated values of the parameters $\bar{a}_{i},(i=1, \ldots, 4)$, as well as the arithmetic mean of the estimated values of the parameters $\bar{a}_{0}, \bar{a}_{0}^{\prime}$, are used as initial guesses for a least-square best-fitting of $H(\boldsymbol{n})$ with a rotated elliptical Lorentzian function superimposed on a linearly varying background

$$
L(\boldsymbol{x})=a_{0}+a_{1} x_{1}+a_{2} x_{2}+\frac{a_{3}}{1+\left(\frac{x_{1}^{\prime}}{a_{4}}\right)^{2}+\left(\frac{\left.x_{2}^{\prime}\right)}{a_{5}}\right)^{2}},
$$

where $x_{1}^{\prime}=\cos \theta x_{1}+\sin \theta x_{2}, x_{2}^{\prime}=-\sin \theta x_{1}+\cos \theta x_{2}$. The value of $\bar{a}_{4}$ is the initial guess of both $a_{4}$ and $a_{5}$, while the initial guess of the rotation angle $\theta$ is zero.

- The extracted image $H(\boldsymbol{n})$ is merged into the sampled function $L(\boldsymbol{n})$, defined on the full image domain, by replacing the values of $L(\boldsymbol{n})$ with the values of $H(\boldsymbol{n})$ in the pixels of the extraction domain. Finally the linearly varying background is subtracted and the result is normalized to unit volume.

If several stars are extracted from the same image, then we take the median of all the results of the previous procedure, and this is normalized again to unit volume, providing the PSF to be used in image deconvolution.

For testing this method we performed a numerical experiment using PSFs generated by the code LOST (Layer Oriented Simulation Tool; Arcidiacono et al. 2004), which is a numerical tool implemented for the simulation of multi conjugate adaptive optics (MCAO) systems using the layer oriented approach. In particular this code is the only one able to simulate the multiple field of view (MFoV) (Ragazzoni et al. 2002) MCAO technique as it will be implemented on the LN instrument. Moreover, it is able to combine the two adaptive optics arms retrieving an adaptive optics corrected LBT-interferometric PSF. The LN instrument configuration has been adopted to generate the PSFs used in the exercise presented in this paper. Because of the lack of information about the turbulence profile above Mount Graham, we adopted the median atmospheric condition of Paranal, another well-studied astronomical site, characterised by an overall 0.71 arcsec seeing in $V$ band, which is not far from recent seeing measurement by a site-testing campaign retrieving a $0.67^{\prime \prime} \pm 0.17^{\prime \prime}$ (Egner et al. 2006).

As reference stars for the adaptive optics system, we used an existing constellation (see Fig. 4) observed during the meridian passage, when the airmass is lower $(\approx 1)$. One major problem of end-to-end simulation tools, such as LOST, is the maximum time exposure, which cannot be longer than a few seconds. This is small amount time interval compared to the correlation time of the speckle pattern, not enough to obtain speckle-free PSF at the very high dynamic range needed to simulate astronomical cases: we averaged PSFs computed by several simulations run using exactly the same input parameters both for the instrument and the atmosphere, but with different phase screens realisations (10 cases) in order to simulate a much longer exposure time than the $8 \mathrm{~s}$ actually done. The monochromatic PSFs
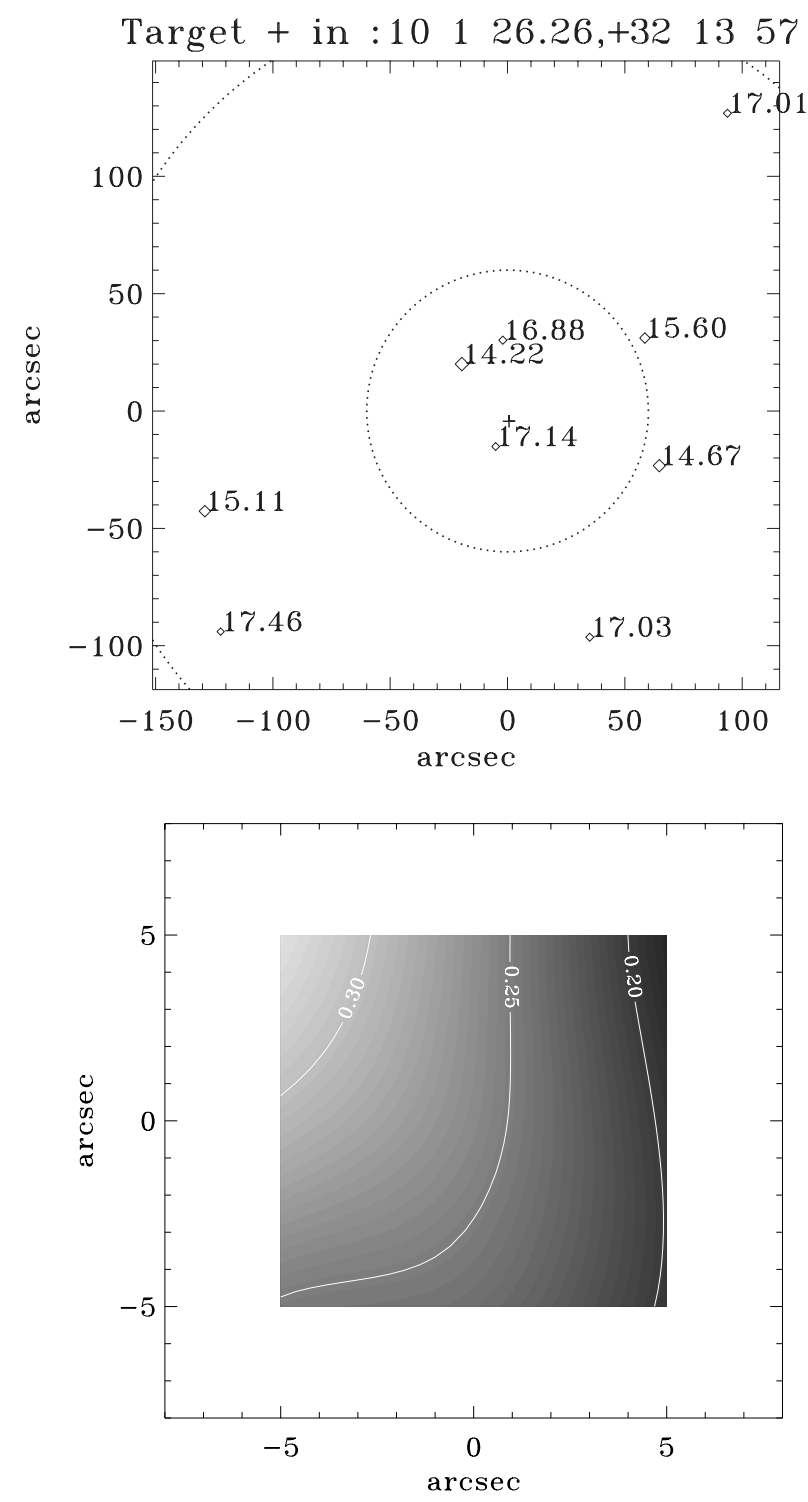

Fig. 4. Upper panel: the reference guide stars used for the simulation of the MCAO LN instrument. The inner circle draws a 2 arcmin FoV (with the stars for high layer correction), while the bigger one is the 6 arcmin FoV (with the stars for ground layer correction). Lower panel: longexposure SR map simulated over the LN infrared camera scientific FoV.

(at $2.13 \mu \mathrm{m}$ ) were shrunk and co-added in order to simulate the bandwidth $(0.02 \mu \mathrm{m})$ corresponding to the $2.12 \mu \mathrm{m}$ filter to be mounted aboard LN. The final platescale matches the LN camera $0.005^{\prime \prime} / \mathrm{px}$. As final output we obtained the $3 \times 3$ PSFs 5 arcsec spaced for the whole $\approx 10^{\prime \prime} \times 10^{\prime \prime}$ LN scientific FoV.

Next, we generated a test object by producing a $384 \times 384$ FITS file of the image of the galaxy NGC 6946 as provided by the second light of LBT (http://medusa.as.arizona.edu/ lbto/astronomical.htm). This file, after background subtraction, was immersed, by zero padding, into a $512 \times 512$ array, and a reference star of magnitude 13 in $K$ s band was added in the pixel $[316,446]$, the integrated magnitude of the galaxy being set to 10 . Next a set of six equispaced images was generated by convolving the six rotated objects with PSFs, computed by LOST, corresponding to the band, mentioned above, with central wavelength $\lambda=2.12 \mu \mathrm{m}$ and width $\Delta \lambda=0.02 \mu \mathrm{m}$. The SR of the PSFs varied from 23.5 to $32.5 \%$. Moreover, for each image we 

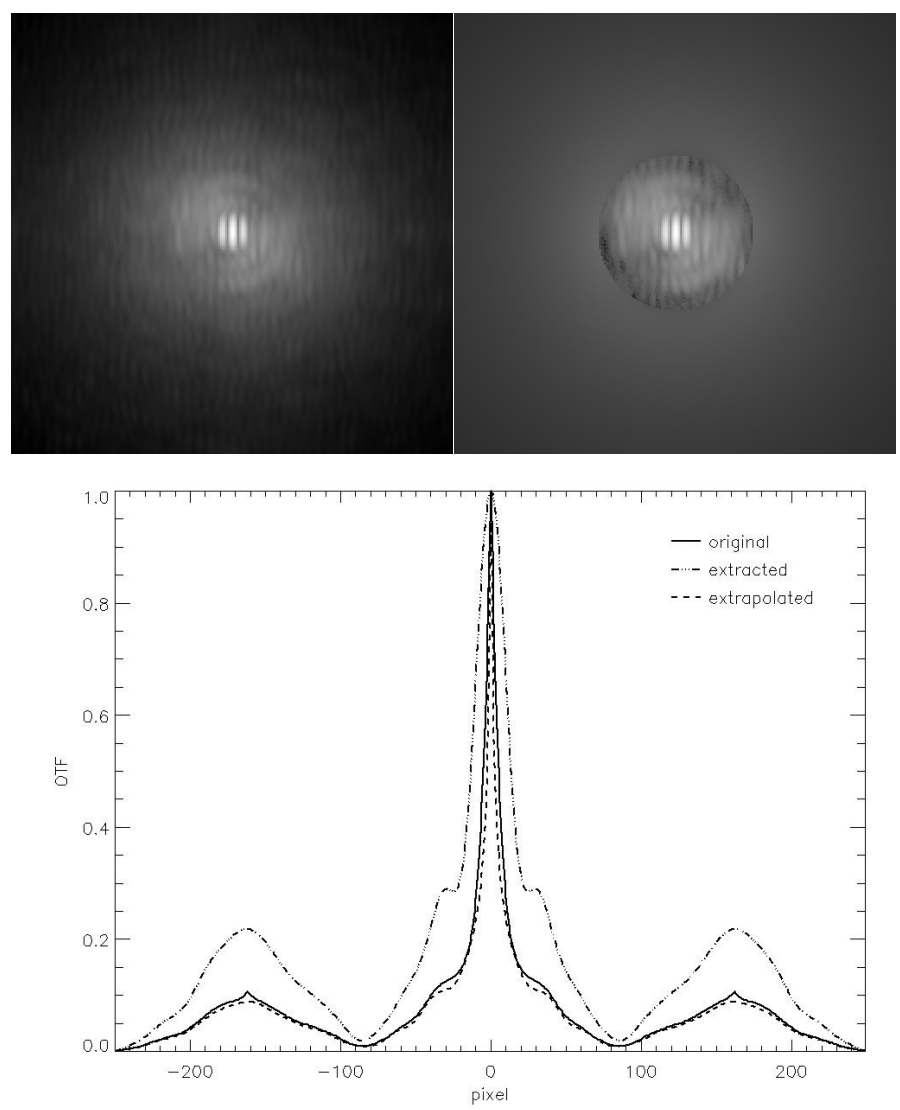

Fig. 5. Example of PSF extraction. Upper panels: the PSF used for generating the image (left panel) and the PSF extracted and extrapolated (right panel). Lower panel: for each PSF we plot the cut, along the baseline, of the corresponding MTF. Full line: the original PSF; dashdotted line: the extracted PSF, with an extraction radius of 45 pixels, extrapolated by zero-padding; dashed line: the PSF extrapolated with the procedure described in the text. The agreement of its MTF with that of the original PSF is fairly good.

assumed a sky background of $13.5 \mathrm{mag} / \operatorname{arcsec}^{2}$ and an integration time of $30 \mathrm{~min}$, while the values of all the other parameters coincide with those of the simulation of Sect. 2.

The six images were counter-rotated by the corresponding angles, as explained in Sect. 2, and we obtained a PSF from each image by extracting the image of the reference star, with an extraction radius $R=45$ pixels and applying the procedure outlined above.

In Fig. 5 we show the result obtained in the case of the image corresponding to $0^{\circ}$. In the upper-left panel we give the PSF used for generating the image (by visualizing the 0.1 power of the image), while we give the extracted and extrapolated PSF in the upper-right panel. The extraction domain is made visible by the small discontinuities due to the merging procedure. In the lower panel the comparison between the PSFs was obtained by plotting the cut along the baseline of the MTF of the original PSF, of the extracted one extrapolated by zero padding, and of that obtained by a Lorentzian extrapolation. The blurring effect due to the finite size of the extraction window is clearly visible, as is the beneficial effect introduced by the Lorentzian extrapolation.

To evaluate the error introduced by the PSF extraction, we first performed the reconstruction of the object using the PSFs obtained by rotating those used for generating the images (an almost "inverse crime" experiment). We obtained a minimum
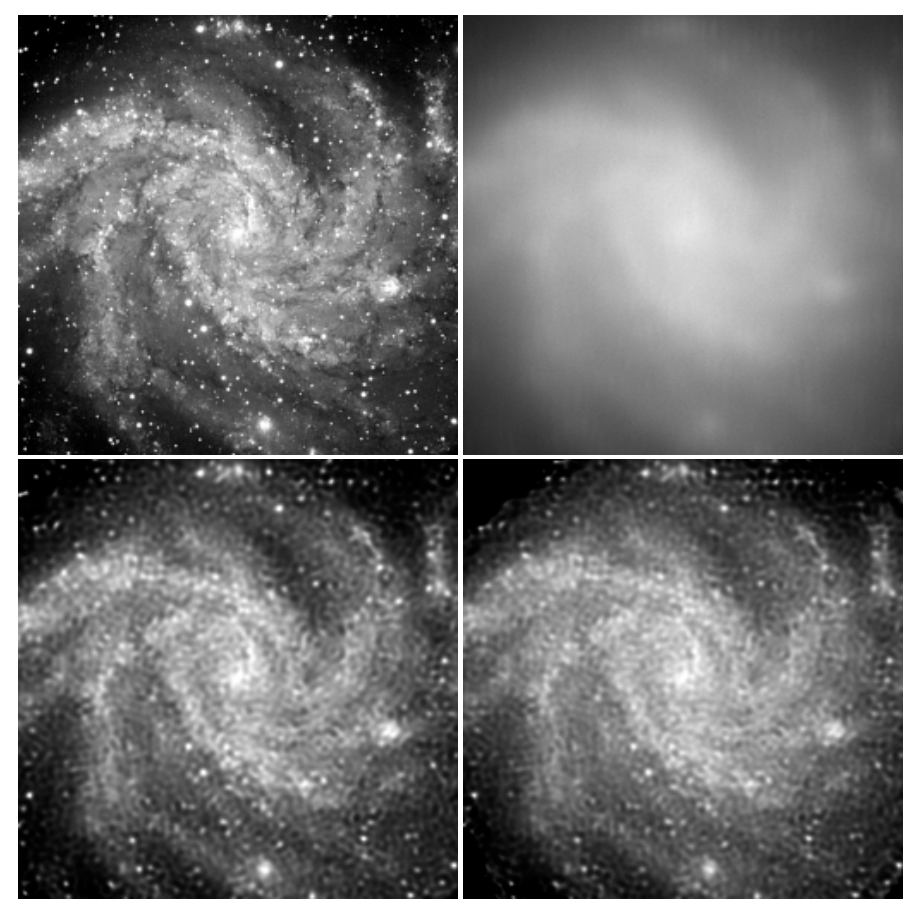

Fig. 6. Upper-left panel: the object to be reconstructed, i.e. the image of the galaxy NGC 6946. Upper-right panel: the image corresponding to $0^{\circ}$. Lower-left panel: the "inverse crime" reconstruction. Lower-right panel: the reconstruction obtained with the extracted PSFs.

relative rms error of about $16 \%$ after 1200 iterations (note that the iterations are reduced to 100 by the two implemented acceleration methods, without a significant change in the reconstruction error). Finally the same images were deconvolved using the extracted PSFs. A minimum reconstruction error of about $18 \%$ was reached after 960 iterations (reduced to 90 by the acceleration methods). Therefore we deduce that an additional $2 \%$ error is introduced by the imperfect knowledge of the PSFs, a result that looks satisfactory.

\section{Concluding remarks}

The main purpose of this paper is to provide methods and algorithms that can be used in the deconvolution of the images of Fizeau interferometers such as LINC-NIRVANA. In particular the OSEM algorithm proposed in Bertero \& Boccacci (2000) is modified in order to compensate for the rotation of the object in the FoV of the camera during the observation process. The method, that is also able to compensate for possible boundary effects, is computationally expensive because it requires the immersion of the rotated images into broader arrays (by zero padding).

Due to the small-angle derotator available on LINC-NIRVANA, it may be necessary to peform several short exposures of the same target to reduce smearing effects on the PSFs; in such a case, deconvolution of a large number of images of the same target is required. At first glance this seems to increase the computational cost of image reconstruction. However, a nice property of OSEM must be taken into account, pointed out by Hudson \& Larkin (1994) in tomography and verified in our applications to LINC-NIRVANA: if we increase the number of images by a factor $q$ (so that the cost of one iteration is also increased by $q$ ), then the number of iterations is reduced by a factor $q$ so that the computational time is 
approximately independent of the number of images. It will essentially depend on the SNR and will be smaller for lower SNR.

In any case, the computational cost may be excessive in many circumstances and, for this reason, we have extended the acceleration methods proposed by Biggs \& Andrews (1997, 1998) to OSEM. The gain we obtain in such a way is interesting. The problem is that the minimum of the reconstruction error is reached quickly, but this error also quickly increases after the minimum, so that a problem arises of stopping the iterations at the appropriate point. As far as we know, no automatic criterion exists that can be applied to the deconvolution of real images. The trivial approach consists in selecting the best result by visual inspection of the iterates. It is obvious that this problem deserves further investigation.

Finally we have developed a method for estimating the PSF from the image of a reference star contained in the FoV. Since the extracted image is defined on a much smaller domain than that of the complete image, an extrapolation is necessary to avoid a "blurring" effect in the $u, v$-plane. We found that an extrapolation in terms of a (in general rotated and elliptical) Lorentzian function can provide satisfactory results. More numerical tests are necessary, and extrapolation in terms of other functions should also be considered.

In conclusion, a processing of $\mathrm{LN}$-images based on the methods proposed in this paper will require the following steps:

- de-rotation of the detected images by means of a de-rotation module of AIRY;
- extraction of the PSFs by means of a corresponding module of AIRY;

- deconvolution by means of the algorithm proposed in this paper (or of an accelerated version of it).

Work is in progress for an accurate validation of this approach.

\section{References}

Anconelli, B., Bertero, M., Boccacci, P., \& Carbillet, M. 2005, A\&A, 430, 731 Anconelli, B., Bertero, M., Boccacci, P., Carbillet, W., \& Lanteri, H. 2006, A\&A, 448,1217

Arcidiacono, C., Diolaiti, E., Tordi, M., et al. 2004, Appl. Opt., 43, 4288

Bertero, M., \& Boccacci, P. 2000, A\&AS, 144, 181

Bertero, M., \& Boccacci, P. 2005, A\&A, 437, 369

Bertero, M., Anconelli, B., Boccacci, P., et al. 2006, in EAS Publ. Series 22, ed. M. Carbillet, A. Ferrari, \& C. Aime, 35

Biggs, D. S. C., \& Andrews 1997, Appl. Opt., 36, 1766

Biggs, D. S. C., \& Andrews 1998, Proc. SPIE, 3461, 33

Carbillet, M., Correia, S., Boccacci, P., \& Bertero, M. 2002, A\&A, 387, 744

Correia, S., Carbillet, M., Boccacci, P., Bertero, M., \& Fini, L. 2002, A\&A, 387, 733

Desiderà, G., Anconelli, B., Bertero, M., Boccacci, P., \& Carbillet, M. 2006, A\&A, 452, 727

Egner, S. E., Masciadri, E., McKenna, D., Herbst, T. M., \& Gaessler, W. 2006, Proc. SPIE, 6272, 257

Hofmann, K. H., Driebe, T., Heininger, M., Scertl, D., \& Weigelt, G. 2005, A\&A, 444, 983

Hudson, H. M., \& Larkin, R. S. 1994, IEEE Trans. Med. Imag., 13, 601

Iusem, A. N. 1991, Math. Meth. Appl. Sci., 14, 573

Lanteri, H., Roche, M., \& Aime, C. 2002, Inverse Problems, 18, 1397

Ragazzoni, R., Diolaiti, E., Farinato, J., et al. 2002, A\&A, 396, 731 